\section{Biometrics: still much too unreliable for everyday use}

SIR - Anil K. Jain gives a misleading picture of the effectiveness of biometric technologies, which identify people on the basis of physical features (News \& Views Q\&A 'Biometric recognition' Nature 449, 38-40; 2007).

Jain says that, since the inception of the US Visitor and Immigrant Status Indicator Technology (US-VISIT) in January 2004, more than 75 million visitors have been processed through it and about 1,000 have been denied entry. According to the US Department of Justice's analysis of US-VISIT after its first year of operation (www.usdoj. gov/oig/reports/plus/e0501/exec.htm), on average 118,000 people pass through each day. Of these, 22,350 are subject to secondary inspection and 1,811 of these are refused entry - considerably more than Jain states.

Of those who are subject to secondary inspection, $92 \%$ are subsequently admitted to the United States. These figures raise doubts about the reliability of the biometric systems used for primary inspections.

Applications such as US-VISIT need to reject virtually all impostors, and keeping the false accept rate (FAR) close to zero pushes the false reject rate (FRR) up. Jain states that current fingerprint-recognition systems can provide a FRR of up to $0.01 \%$ at a FAR of $0.1 \%$. However, in a 2005 UK Passport Service trial (see http://dematerialisedid.com/PDFs/ UKPSBiometrics_Enrolment_Trial_Report. pdf), the FRR for fingerprints was $19 \%$.

One objective of the proposed UK National Identity Scheme is to make it easier for people to prove their identity. At current performance, biometrics based on fingerprints could instead make it harder for nearly a fifth of the population to prove that they have the right to work in the United Kingdom and enjoy social entitlements.

\section{David Moss}

Business Consultancy Services Ltd,

58 Vineyard Hill Road, London SW19 7JH, UK

http://dematerialisedid.com

\section{Biometrics: easy to steal, hard to regain identity}

SIR - In your News \& Views Q\&A

'Biometric recognition' (Nature 449, 38-40;

2007), Anil K. Jain asserts that biometric

technologies are more difficult to abuse than traditional methods of identification.

However, we all leave a biometric trail in our daily lives: our fingerprints on a drinking glass, our voice on a telephone answering machine, our iris patterns on a photograph. We have little ability to change such characteristics and little control over this trail, which makes biometrics useful to forensic science.
But it's exactly these properties that make biometrics a poor replacement for passwords and ID cards, since it's easy for an intruder to collect someone's fingerprint or iris scan without their knowledge, and then inject it into a biometric identification system. Even if the victim becomes aware of the problem, it's impossible to revoke the biometric. If your credit card is stolen, the card company can send you a new one with a different number, but you can't get a new set of fingerprints.

It is precisely because biometric information is irrevocable and unwittingly provided in our daily lives that it is so useful to organizations that regulate the individual (for example, the US Immigration Service), but of little use where the individual controls identification and authorization.

Andrew Watson

72 Kimberley Road, Cambridge CB4 1HJ, UK

\section{Organic synthesis remains relevant in drug discovery}

SIR - As you point out in your News story 'Chemists synthesize a natural-born killer' (Nature 448, 630-631; 2007), some researchers question the merits of organic synthesis, whereby chemists seek to recreate a synthetic version of a natural product. In its defence, I want to remind readers that when Elias James Corey received a Nobel prize in 1990 for the development of organic synthesis, the press release stated: "To perform the total syntheses successfully, Corey was also obliged to develop some fifty entirely new or considerably improved synthesis reactions or reagents... which... have become commonplace in the synthesizing laboratory."

Such newly discovered reactions and reagents are routinely used in the discovery of drug candidates. The cost of pharmaceuticals is, to some extent, directly related to the ease or complexity of their synthesis, and there are still many drugs in the pipeline whose development has been hindered by problems encountered during their manufacture.

This underlines the point that the science of organic synthesis is still a worthy cause to be pursued.

\section{Mukund M. Mehrotra}

Portola Pharmaceuticals, 270 East Grand Avenue, South San Francisco, California 94080, USA

\section{Ocean-drilling vessel should soon be afloat again}

SIR - Those involved in the Integrated Ocean Drilling Program (IODP) are delighted by your encouragement of their work in your Editorial 'Drill often, drill deep' (Nature 449, 260; 2007) and News Feature
'Staying afloat' (Nature 449, 280; 2007).

However, it is not correct to state that the IODP's US platform, the JOIDES Resolution, has languished at a shipyard since 2003. This research vessel completed 10 expeditions between June 2004 and December 2005, each with a full complement of scientists from the United States, Europe and Japan.

The JOIDES Resolution has been in a Singapore shipyard since September 2006, undergoing a complete overhaul. Work has continued since it entered the shipyard, although the hostile business climate described in your Editorial has caused a few delays, as sky-high oil prices have meant higher costs and increased pressures for time in the yard. Interested readers can track progress via pictures of the overhaul at www.joiscience.org/SODV/status.

The JOIDES Resolution is scheduled to resume drilling activities in spring 2008. The shipyard schedule, weather conditions and Japanese fishing-fleet restrictions will not allow it to join its sister ship, the Japanese Chikyu, in 2008. But the JOIDES Resolution will be an active participant in NanTroSEIZE, the Nankai Trough Seismogenic Zone Experiment, by 2010.

Steve Bohlen

Joint Oceanographic Institutions,

1201 New York Avenue NW,

Washington DC 20005, USA

\section{Sic is not enough; historical wording should be changed}

SIR - Your decision to insert sic after "scientific men" in reprints of your historical mission statement ('Men [sic]' Nature 448, $728 ; 2007)$ shows that you are more concerned about the historical integrity of your mission statement than for decency and justice. Why make a mere "tiny step" in the "right direction"? A simple addition of "... and women" would have brought you to the goal.

Isn't it enough that even such a "tiny step" took Nature nearly 140 years? Pious traditionalism is being used to excuse prejudices in many quarters, but it is shocking to encounter it in a supposed bastion of enlightenment and intellectual progress. Do not consider yourself excused. Sanya Samac

Imaging Research, Sunnybrook Research Institute, 2075 Bayview Avenue, North York,

Ontario M4N 3M5, Canada

The Editorial related only to reprinting of our historical 1869 mission statement, not to our current statement (www.nature. com/nature/about/index.html), which uses the term 'scientists'. Comments are welcome at Nautilus, http://blogs.nature. com/nautilus/2007/08/women_and_men_ of_science.html - Editor, Nature. 